\title{
Synthesis and Structural Elucidation of Some Novel Penta-Coordinated Mono-Di-Ethylaluminium(III) Complexes with Internally Functionalized Oximes
}

\author{
Amit K. Jain, Rakesh Bohra* and Ram C. Mehrotra \\ Department of Chemistry, University of Rajasthan, Jaipur-302004, India \\ rkbohra@satyam.net.in
}

\begin{abstract}
Reactions of triethylaluminium etherate adduct $\left(\mathrm{Et}_{3} \mathrm{Al} \mathrm{Et}_{2} \mathrm{O}\right)$ with internally functionalized oximes $\{\operatorname{Ar}(\mathrm{R}) \mathrm{C}=\mathrm{NOH}\}$ in $1: 1$ and $1: 2$ molar ratios in diethyl ether yielded mono- and di-ethylaluminium(III) complexes of the types $\mathrm{Et}_{2} \mathrm{Al}\left\{\mathrm{ON}=\mathrm{C}(\mathrm{R}) \mathrm{Ar}\right.$ ) and $\mathrm{EtAl}\left\{\mathrm{ON}=\mathrm{C}(\mathrm{R}) \mathrm{Ar}_{2}\right\}_{2}$ (where $\mathrm{R}=\mathrm{CH}_{3}$ or $\mathrm{H}$ and $\mathrm{Ar}=\mathrm{C}_{5} \mathrm{H}_{4} \mathrm{~N}$ $2, \mathrm{C}_{4} \mathrm{H}_{3} \mathrm{~S}-2, \mathrm{C}_{4} \mathrm{H}_{3} \mathrm{O}-2$ ). All the above complexes are soluble in common organic solvents, which have been characterized by elemental analysis, IR and multinuclear $\mathrm{NMR}\left({ }^{1} \mathrm{H},{ }^{13} \mathrm{C}\right.$ and $\left.{ }^{27} \mathrm{Al}\right)$. Spectral studies. The ${ }^{27} \mathrm{Al}$ NMR chemical shift values indicate penta-coordination around aluminium(III) atom in both the above types of complexes. Molecular weight measurements in refluxing benzene exhibit monomeric and dimeric nature for the monoethyl and the diethyl complexes, respectively, which gets further support from the FAB mass

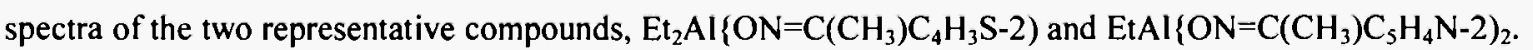

\section{INTRODUCTION}

Organoaluminium compounds are used widely as reagents in organic synthesis and as catalysts in many synthetic processes $/ 1,2 /$. For a better understanding of their role in synthesis and catalysis, it is essential to know the coordination ability of aluminium in such types of derivatives. Generally coordination numbers four and six are more common $/ 3-7 /$ than five /8-10/ in such compounds.

It has also been shown recently $/ 11-16 /$ that aluminium atoms in binuclear metallo-rganic complexes usualy acquire unsymmetrical structures containing aluminium(III) atoms in four- and six-coordination states rather than a symmetrical five-cooordinated structure $/ 13 \%$.

In view of the increasing interest in penta-coordinated organoaluminium(III) complexes $/ 8-10 /$, we have also reported probably the first known example of a five-coordinated heteroleptic metallo-organic compound of $\mathrm{Al}(\mathrm{III}):\left[\left\{\mathrm{C}_{6} \mathrm{H}_{4} \mathrm{O}\left(\mathrm{CH}=\mathrm{NC}_{6} \mathrm{H}_{5}\right)\right\}_{2} \mathrm{AI}\left\{\mathrm{ON}=\mathrm{C}\left(\mathrm{CH}_{3}\right) \mathrm{C}_{4} \mathrm{H}_{3} \mathrm{~S}-2\right\}\right] / 17 \%$.

Further, amongst multidentate organic ligands, oximes have been known to have biological functions / 18 $20 /$ such as growth regulatory, antimicrobial and fungicidal activities. The oxime groups are also present in the althiomycin antibiotic molecule $/ 21 \%$. 
In view of the above, we report here the chemistry of a series of ethylaluminium(III) complexes with internally functionalized oximes.

\section{MATERIALS AND METHODS}

All the experimental manipulations were carried out under strictly inert conditions in anhydrous argon atmosphere. The solvents and reagents were purified by conventional techniques. $\mathrm{The} \mathrm{Et}_{3} \mathrm{AI}_{\mathrm{I}} \mathrm{Et}_{2} \mathrm{O} / 22 /$ and oximes $/ 23 /$ were synthesized and purified according to literature methods. Aluminium was estimated gravimetrically as oxinate /24/. Microanalyses were carried out on a Perkin Elmer-C, H, N \& S II series analyzer. The IR spectra were recorded as Nujol mull on a Nicolet Magna-550 spectrometra in the range $4000-400 \mathrm{~cm}^{-}$ ${ }^{1}$, ${ }^{1} \mathrm{H} .{ }^{13} \mathrm{C}$ and ${ }^{27} \mathrm{AI} \mathrm{NMR}$ spectra were recorded in $5 \mathrm{~mm} \mathrm{NMR}$ tubes as freshly prepared $\mathrm{CDCl}_{3}$ solutions on a Bruker DPX-300 spectrometer operating at $300,75.5$ and $111.9 \mathrm{MHz}$, respectively. Spectra were references with internal chloroform peak $\left({ }^{*} 7.26 \mathrm{ppm}\right.$ for ${ }^{1} \mathrm{H}$ and $77.0 \mathrm{ppm}$ for $\left.{ }^{13} \mathrm{C}\right)$. FAB mass spectra were recorded on a Jeol SX 102/DA-6000 mass spectrometer/data system using argon/xenon (6 KV, 10MA) as the FAB gas, m-nitrobenzyl alcohol was used as the matrix. Molecular weight measurements were carried out by elevation in boiling point method, using a Beckmann's Thermometer fitted in a glass assembly in anhydrous benzene. the instrument was calibrated using samples of known molecular weight like naphthalene/benzophenone/ benzil in anhydrous benzene $\left(M=1000 K_{b} \cdot w / \Delta T_{b} \cdot W\right.$, where $M=$ molecular weight, $w=$ weight of solute in gms, $K_{b}=$ molar elevation constant, $W=$ weight of solvents in gms, $\Delta T_{b}=$ elevation in boiling point).

\section{Preparation of $\mathrm{Et}_{2} \mathrm{Al}\left\{\mathrm{ON}=\mathrm{C}\left(\mathrm{CH}_{3}\right) \mathrm{C}_{5} \mathrm{H}_{4} \mathrm{~N}-2\right)$}

A solution of $\mathrm{Et}_{3} \mathrm{Al}_{\text {. }} \mathrm{Et}_{2} \mathrm{O}(1.75 \mathrm{~g}, 9.32 \mathrm{mmol})$ in $20 \mathrm{ml}$ of anhydrous diethyl ether was slowly added to an etheral solution of $\mathrm{HON}=\mathrm{C}\left(\mathrm{CH}_{3}\right) \mathrm{C}_{5} \mathrm{H}_{4} \mathrm{~N}-2(1.27 \mathrm{gm}, 9.32 \mathrm{mmol})$ under argon atmosphere. The reaction mixture was stirred at room temperature for $4 \mathrm{~h}$. After stirring, the solvents were evaporated under vacuum and dried to give a yellow-coloured paste. The product was washed $(2 \times 10 \mathrm{ml})$ with petroleum ether. Further, it was dried in vacuum to afford a yellow solid (yield $98 \%$ ). This was recrystallized from a mixture of dichloromethane and $n$-hexane (7:1).

All other diethylaluminium(III) complexes $(3,5,7$ and 9$)$ were prepared using a similar route. Their synthetic details and analytical data are summarized in Table 1.

\section{Preparation of EtAl $\left\{\mathrm{ON}=\mathrm{C}\left(\mathrm{CH}_{3}\right) \mathrm{C}_{5} \mathrm{H}_{4} \mathrm{~N}-2\right)$}

An etheral solution of $\mathrm{Et}_{3} \mathrm{AI}_{\mathrm{Et}} \mathrm{Ot}(1.29 \mathrm{~g}, 6.85 \mathrm{mmol})$ was gradually added to another etheral solution of $\mathrm{HON}=\mathrm{C}\left(\mathrm{CH}_{3}\right) \mathrm{C}_{5} \mathrm{H}_{4} \mathrm{~N}-2(1.87 \mathrm{gm}, 13.7 \mathrm{mmol})$ under argon atmosphere. The contents were allowed to remain at room temperature for $4 \mathrm{~h}$. After removal of the solvent under reduced pressure, the product obtained was washed $(2 \times 10 \mathrm{ml})$ with $\mathrm{n}$-hexane. Further it was dried under vacuum to afford a yellow solid (yield $98 \%$ ).

The product obtained was recrystallized from a mixture of dichloromethane and $n$-hexane (7:1). 
Table I

Synthetic and analytical data of ethylaluminium(III) complexes

\begin{tabular}{|c|c|c|c|c|c|c|c|c|c|}
\hline \multirow[t]{2}{*}{$\begin{array}{l}\text { S. } \\
\text { No. }\end{array}$} & \multirow[t]{2}{*}{ Complexes } & \multirow[t]{2}{*}{$\begin{array}{l}\text { Yield } \\
(\%)\end{array}$} & \multirow{2}{*}{$\begin{array}{l}\text { Colour } \\
\text { and } \\
\text { State }\end{array}$} & \multicolumn{4}{|c|}{$\begin{array}{l}\text { Elemental analysis ( \% ) } \\
\text { Found (Calcd.) }\end{array}$} & \multirow{2}{*}{$\begin{array}{l}\text { M.Wt. } \\
\text { Found } \\
\text { (Calcd.) }\end{array}$} & \multirow[t]{2}{*}{$\begin{array}{l}\text { M.P. } \\
\left({ }^{\circ} \mathrm{C}\right)\end{array}$} \\
\hline & & & & C & $\mathrm{H}$ & $\mathbf{N}$ & $\mathrm{Al}$ & & \\
\hline 1. & $\mathrm{Et}_{2} \mathrm{Al}\left\{\mathrm{ON}=\mathrm{C}(\mathrm{Me}) \mathrm{C}_{5} \mathrm{H}_{4} \mathrm{~N}-2\right\}$ & 98 & $\begin{array}{l}\text { Yellow } \\
\text { solid }\end{array}$ & $\begin{array}{c}58.8 \\
(60.0)\end{array}$ & $\begin{array}{c}7.75 \\
(7.78)\end{array}$ & $\begin{array}{c}12.5 \\
(12.7)\end{array}$ & $\begin{array}{c}12.0 \\
(12.3)\end{array}$ & $\begin{array}{c}447 \\
(220)\end{array}$ & $\begin{array}{l}242 \\
\text { dec. }\end{array}$ \\
\hline 2. & $\mathrm{EtAl}\left\{\mathrm{ON}=\mathrm{C}(\mathrm{Me}) \mathrm{C}_{3} \mathrm{H}_{4} \mathrm{~N}-2\right\}_{2}$ & 98 & $\begin{array}{l}\text { Yellow } \\
\text { Solid } \\
\end{array}$ & $\begin{array}{l}58.4 \\
(58.9)\end{array}$ & $\begin{array}{c}5.84 \\
(5.87)\end{array}$ & $\begin{array}{c}17.0 \\
(17.2)\end{array}$ & $\begin{array}{c}8.24 \\
(8.27)\end{array}$ & $\begin{array}{c}320 \\
(326)\end{array}$ & 218 \\
\hline 3. & $\mathrm{Et}_{2} \mathrm{Al}\left\{\mathrm{ON}=\mathrm{C}(\mathrm{Me}) \mathrm{C}_{4} \mathrm{H}_{3} \mathrm{~S}-2\right\}$ & 93 & \begin{tabular}{|l|} 
Yellow \\
Solid \\
\end{tabular} & $\begin{array}{c}53.0 \\
(53.3)\end{array}$ & $\begin{array}{c}7.10 \\
(7.16)\end{array}$ & $\begin{array}{c}6.18 \\
(6.22)\end{array}$ & $\begin{array}{r}11.9 \\
(12.0)\end{array}$ & $\begin{array}{c}460 \\
(225)\end{array}$ & $\begin{array}{l}148 \\
\text { dec. }\end{array}$ \\
\hline 4. & $\overline{E t A l}\left\{\mathrm{ON}=\mathrm{C}(\mathrm{Me}) \mathrm{C}_{4} \mathrm{H}_{3} \mathrm{~S}-2\right\}_{2}$ & 93 & $\begin{array}{l}\text { Cream } \\
\text { Solid } \\
\end{array}$ & $\begin{array}{c}49.4 \\
(49.9) \\
\end{array}$ & $\begin{array}{c}5.00 \\
(5.09) \\
\end{array}$ & $\begin{array}{c}8.18 \\
(8.33) \\
\end{array}$ & $\begin{array}{c}8.00 \\
(8.03) \\
\end{array}$ & $\begin{array}{c}328 \\
(336) \\
\end{array}$ & 135 \\
\hline 5. & $\mathrm{Et}_{2} \mathrm{Al}\left\{\mathrm{ON}=\mathrm{C}(\mathrm{Me}) \mathrm{C}_{4} \mathrm{H}_{3} \mathrm{O}-2\right\}$ & 96 & \begin{tabular}{|l|} 
Yellow \\
Solid \\
\end{tabular} & $\begin{array}{r}57.0 \\
(57.4) \\
\end{array}$ & $\begin{array}{r}7.68 \\
(7.71) \\
\end{array}$ & $\begin{array}{r}6.67 \\
(6.69) \\
\end{array}$ & $\begin{array}{c}12.8 \\
(12.9) \\
\end{array}$ & $\begin{array}{c}422 \\
(209) \\
\end{array}$ & $\begin{array}{l}192 \\
\text { dec. }\end{array}$ \\
\hline 6. & $\mathrm{EtAl}\left\{\mathrm{ON}=\mathrm{C}(\mathrm{Me}) \mathrm{C}_{4} \mathrm{H}_{3} \mathrm{O}-2\right\}_{2}$ & 95 & $\begin{array}{l}\text { Brown } \\
\text { Solid }\end{array}$ & $\begin{array}{c}55.1 \\
(55.3)\end{array}$ & $\begin{array}{c}5.60 \\
(5.63) \\
\end{array}$ & $\begin{array}{c}9.06 \\
(9.21)\end{array}$ & $\begin{array}{l}8.81 \\
(8.87)\end{array}$ & $\begin{array}{c}296 \\
(304)\end{array}$ & 130 \\
\hline 7. & $\mathrm{Et}_{2} \mathrm{Al}\left\{\mathrm{ON}=\mathrm{CH}\left(\mathrm{C}_{4} \mathrm{H}_{3} \mathrm{~S}-2\right)\right\}$ & 96 & $\begin{array}{l}\text { White } \\
\text { Solid }\end{array}$ & $\begin{array}{c}50.8 \\
(51.2)\end{array}$ & $\begin{array}{c}6.62 \\
(6.68)\end{array}$ & $\begin{array}{c}6.60 \\
(6.63)\end{array}$ & $\begin{array}{l}12.8 \\
(12.8)\end{array}$ & $\begin{array}{l}418 \\
(211)\end{array}$ & $\begin{array}{l}252 \\
\text { dec. }\end{array}$ \\
\hline 8. & $\mathrm{EtAl}\left\{\mathrm{ON}=\mathrm{CH}\left(\mathrm{C}_{4} \mathrm{H}_{3} \mathrm{~S}-2\right)\right\}_{2}$ & 96 & $\begin{array}{l}\text { White } \\
\text { Solid }\end{array}$ & $\begin{array}{c}46.2 \\
(46.7)\end{array}$ & $\begin{array}{c}4.21 \\
(4.25)\end{array}$ & $\begin{array}{c}9.00 \\
(9.08)\end{array}$ & $\begin{array}{c}8.75 \\
(8.77)\end{array}$ & $\begin{array}{c}298 \\
(308)\end{array}$ & $\begin{array}{l}254 \\
\text { dec. }\end{array}$ \\
\hline 9. & $\mathrm{Et}_{2} \mathrm{Al}\left\{\mathrm{ON}=\mathrm{CH}\left(\mathrm{C}_{4} \mathrm{H}_{3} \mathrm{O}-2\right)\right\}$ & 98 & $\begin{array}{l}\text { White } \\
\text { Solid } \\
\end{array}$ & $\begin{array}{c}54.8 \\
(55.4) \\
\end{array}$ & $\begin{array}{c}7.21 \\
(7.23) \\
\end{array}$ & $\begin{array}{r}7.02 \\
(7.18) \\
\end{array}$ & $\begin{array}{c}13.8 \\
(13.8) \\
\end{array}$ & $\begin{array}{c}400 \\
(195) \\
\end{array}$ & $\begin{array}{l}220 \\
\text { dec. }\end{array}$ \\
\hline 10. & $\mathrm{EtAl}\left\{\mathrm{ON}=\mathrm{CH}\left(\mathrm{C}_{4} \mathrm{H}_{3} \mathrm{O}-2\right)\right\}_{2}$ & 98 & $\begin{array}{l}\text { White } \\
\text { Solid }\end{array}$ & $\begin{array}{c}52.0 \\
(52.2) \\
\end{array}$ & $\begin{array}{c}4.70 \\
(4.74) \\
\end{array}$ & $\begin{array}{c}10.1 \\
(10.2)\end{array}$ & $\begin{array}{c}9.75 \\
(9.77)\end{array}$ & $\begin{array}{c}264 \\
(276) \\
\end{array}$ & $\begin{array}{l}234 \\
\text { dec. }\end{array}$ \\
\hline
\end{tabular}

Other remaining monoethylaluminium(III) complexes $(4,6,8$ and 10) were prepared using a similar route. Their synthetic details and analytical data are summarized in Table $\mathrm{I}$.

\section{RESULTS AND DISCUSSION}

All the above reactions were carried out under strictly anhydrous and inert atmosphere. Reactions of $\mathrm{Et}_{3} \mathrm{Al} \mathrm{Et}_{2} \mathrm{O}$ with the corresponding internally functionalized oximes in 1:1 and 1:2 molar ratios in diethyl ether afforded products of the types, $\mathrm{Et}_{2} \mathrm{Al}\{\mathrm{ON}=\mathrm{C}(\mathrm{R}) \mathrm{Ar}\}$ and $\mathrm{EtAl}\{\mathrm{ON}=\mathrm{C}(\mathrm{R}) \mathrm{Ar}\}_{2}$ (where $\mathrm{R}=\mathrm{CH}_{3}$ or $\mathrm{H}$ and $\mathrm{Ar}=\mathrm{C}_{5} \mathrm{H}_{4} \mathrm{~N}-2, \mathrm{C}_{4} \mathrm{H}_{3} \mathrm{~S}=2, \mathrm{C}_{4} \mathrm{H}_{3} \mathrm{O}-2$ ). The general reaction may be illustrated schematically as:

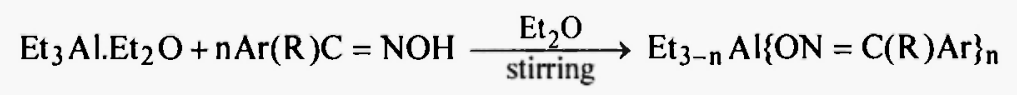

(where $\mathrm{R}=\mathrm{CH}_{3}$ or $\mathrm{H}$ and $\mathrm{Ar}=\mathrm{C}_{5} \mathrm{H}_{4} \mathrm{~N}-2, \mathrm{C}_{4} \mathrm{H}_{3} \mathrm{~S}-2, \mathrm{C}_{4} \mathrm{H}_{3} \mathrm{O}-2, \mathrm{n}=1$ or 2 )

All the above complexes are yellow, brown or white solids (Table 1) and are soluble in common organic solvents. These compounds were purified by recrystallization from a mixture of dichloromethane and nhexane. Molecular weight measurements in refluxing benzene exhibit monomeric and dimeric nature for the monoethyl and the diethyl complexes, respectively. 


\section{IR Spectra}

Tentative assignments of some of the important IR bands have been made and are summarized in Table II. Broad stretching vibrations at $-3300 \mathrm{~cm}^{-1}$ due to $-\mathrm{OH}$ group of the free oximes disappeared in the IR spectra of these derivatives, suggesting the deprotonation of $-\mathrm{OH}$ group and formation of $\mathrm{Al}-\mathrm{O}$ bond. Formation of $\mathrm{Al}-\mathrm{O} / 25,26$ / bond is further supported by the appearance of a weak-to-strong intensity band in the region 708-766 $\mathrm{cm}^{-1}$. The appearance of $\mathrm{vN}-\mathrm{O} / 27 /$ and $\mathrm{vC}=\mathrm{N} / 28 /$ stretching vibrations towards lower frequencies $\left(-20 \mathrm{~cm}^{-1}\right)$ further supports the formation of $\mathrm{Al}-\mathrm{O}$ bond. The appearance of $\mathrm{v}(\mathrm{C}=\mathrm{N})$ stretching frequencies at lower wave number, as compared to the free ligand moieties, indicates the coordination of aluminium to the nitrogen atom of the ligand. This was further confirmed by the appearance of a new weakto-strong intensity band in the region $641-700 \mathrm{~cm}^{-1}$, which may be assigned to $\mathrm{v}(\mathrm{Al}-\mathrm{N})$ mode $/ 12,13,16 /$. The weak-to-strong intensity bands in the region $550-616 \mathrm{~cm}^{-1}$ were assigned to $\mathrm{v}(\mathrm{Al}-\mathrm{C})$. The $\mathrm{Al}-\mathrm{O}-\mathrm{Al} / 12,13 / 16$ / vibrations have been observed in the region $766-841 \mathrm{~cm}^{-1}$.

Table 11

Some relevant IR spectral data of ethylaluminium(III) complexes

\begin{tabular}{|c|c|c|c|c|c|c|c|}
\hline \multirow{2}{*}{$\begin{array}{c}\text { S. } \\
\text { No. }\end{array}$} & \multirow[t]{2}{*}{ Compound } & \multicolumn{2}{|c|}{ Oximate moiety } & \multirow[t]{2}{*}{ vAI-C } & \multirow[t]{2}{*}{ vAl-O } & \multirow[t]{2}{*}{ vAl-N } & \multirow[t]{2}{*}{$v A|-O-A|$} \\
\hline & & $\mathrm{vN}-\mathrm{O}$ & $v C=N$ & & & & \\
\hline 1. & $\mathrm{Et}_{2} \mathrm{Al}\left\{\mathrm{ON}=\mathrm{C}(\mathrm{Me}) \mathrm{C}_{3} \mathrm{H}_{4} \mathrm{~N}-2\right\}$ & $916 \mathrm{~m}$ & $1550 \mathrm{~m}$ & $600 \mathrm{~s}$ & $733 \mathrm{~s}$ & $650 \mathrm{~s}$ & $783 \mathrm{~s}$ \\
\hline 2. & $\mathrm{EtAl}\left\{\mathrm{ON}=\mathrm{C}(\mathrm{Me}) \mathrm{C}_{5} \mathrm{H}_{6} \mathrm{~N}-2\right\}_{2}$ & $916 w$ & $1583 w$ & $583 w$ & $766 w$ & $700 \mathrm{~m}$ & $\overline{-}$ \\
\hline 3. & $\mathrm{Et}_{2} \mathrm{Al}\left\{\mathrm{ON}=\mathrm{C}(\mathrm{Me}) \mathrm{C}_{4} \mathrm{H}_{3} \mathrm{~S}-2\right\}$ & $900 \mathrm{~m}$ & $1566 w$ & $583 \mathrm{~m}$ & $716 s$ & $64 \mathrm{Im}$ & $783 m$ \\
\hline 4. & $\mathrm{EtAl}\left\{\mathrm{ON}=\mathrm{C}(\mathrm{Me}) \mathrm{C}_{4} \mathrm{H}_{3} \mathrm{~S}-2\right\}_{2}$ & $883 w$ & $1566 \mathrm{w}$ & $550 w$ & $716 s$ & $675 \mathrm{~m}$ & - \\
\hline 5. & $\mathrm{Et}_{2} \mathrm{Al}\left\{\mathrm{ON}=\mathrm{C}(\mathrm{Me}) \mathrm{C}_{4} \mathrm{H}_{3} \mathrm{O}-2\right\}$ & $891 w$ & $1541 w$ & $600 w$ & $716 \mathrm{~s}$ & $675 w$ & $766 w$ \\
\hline 6. & $\mathrm{EtAl}\left\{\mathrm{ON}=\mathrm{C}(\mathrm{Me}) \mathrm{C}_{4} \mathrm{H}_{3} \mathrm{O}-2\right\}_{2}$ & $900 \mathrm{~s}$ & $1566 \mathrm{~m}$ & $600 \mathrm{~s}$ & $733 \mathrm{vs}$ & $6 \overline{050}$ & $=$ \\
\hline 7. & $\mathrm{Et}_{2} \mathrm{Al}\left\{\mathrm{ON}=\mathrm{CH}\left(\mathrm{C}_{4} \mathrm{H}_{3} \mathrm{~S}-2\right)\right\}$ & $891 \mathrm{vs}$ & $1583 \mathrm{~s}$ & $616 \mathrm{vs}$ & $708 v s$ & $666 \mathrm{vs}$ & $841 v s$ \\
\hline 8. & $\mathrm{EtAl}\left\{\mathrm{ON}=\mathrm{CH}\left(\mathrm{C}_{4} \mathrm{H}_{3} \mathrm{~S}-2\right)\right\}_{2}$ & $883 \mathrm{vs}$ & $1600 \mathrm{~s}$ & $616 \mathrm{vs}$ & $716 \mathrm{vs}$ & $666 \mathrm{vs}$ & - \\
\hline 9. & $\mathrm{Et}_{2} \mathrm{Al}\left\{\mathrm{ON}=\mathrm{CH}\left(\mathrm{C}_{4} \mathrm{H}_{3} \mathrm{O}-2\right)\right\}$ & $875 v s$ & $1591 \mathrm{~s}$ & $600 \mathrm{vs}$ & $750 \mathrm{vs}$ & $650 \mathrm{vs}$ & $800 \mathrm{~s}$ \\
\hline 10. & $\operatorname{EtAl}\left\{\mathrm{ON}=\mathrm{CH}\left(\mathrm{C}_{4} \mathrm{H}_{3} \mathrm{O}=2\right)\right\}_{2}$ & $866 \mathrm{vs}$ & $1600 \mathrm{~s}$ & $583 \mathrm{vs}$ & $733 \mathrm{vs}$ & $675 s$ & - \\
\hline
\end{tabular}

\section{'H NMR Spectra}

The important signals in 'H NMR spectra of these derivatives are summarized in Table III. A comparison of the spectra of the free oximes with the spectra of the corresponding derivatives shows the absence of $-\mathrm{OH}$ signals, indicating deprotonation of the hydroxy group of the oxime ligands and formation of Al-O bond. 
Table IH

NMR $\left({ }^{1} \mathrm{H},{ }^{13} \mathrm{C}\right.$ and $\left.{ }^{27} \mathrm{Al}\right)$ spectral data of ethylaluminium(III) complexes

\begin{tabular}{|c|c|c|c|c|c|}
\hline \multirow{2}{*}{$\begin{array}{l}\text { Com } \\
\text {-pley } \\
\text { No*. }\end{array}$} & \multicolumn{2}{|c|}{${ }^{1} \mathrm{H} N M R$ in $\delta \mathrm{ppm}$} & \multicolumn{2}{|c|}{${ }^{13} \mathrm{C} \mathrm{NMR}$ in $\delta \mathrm{ppm}$} & \multirow{2}{*}{$\begin{array}{l}27 \mathrm{Al} \\
\mathrm{NMR} \\
\text { in } \delta \mathrm{ppm}\end{array}$} \\
\hline & Ethyl moiety & Oximate moiety & Ethyl moiety & Oximate moiety & \\
\hline 1. & $\begin{array}{l}0.06\left(\mathrm{~m}, \mathrm{CH}_{2}\right) \\
1.07\left(\mathrm{t}, \mathrm{CH}_{3}\right)\end{array}$ & $\begin{array}{l}2.12\left(\mathrm{~s}, \mathrm{CH}_{3}\right) ; \\
7.35(\mathrm{t}, \mathrm{H}-4) ; 7.57 \\
(\mathrm{t}, \mathrm{H}-5) ; \\
8.05(\mathrm{~d}, \mathrm{H}-3) ; \\
8.62(\mathrm{br}, \mathrm{H}-6)\end{array}$ & $\begin{array}{l}0.92\left(\mathrm{CH}_{2}\right) ; \\
18.3\left(\mathrm{CH}_{3}\right)\end{array}$ & $\begin{array}{l}9.65\left(\mathrm{CH}_{3}\right) ; 119.7 \\
(\mathrm{C}-5) ; 120.2(\mathrm{C}-3) \\
121.8(\mathrm{C}-4) ; 125.1 \\
(\mathrm{C}-6) ; 140.4(\mathrm{C}-2) \\
148.3(\mathrm{C}=\mathrm{N})\end{array}$ & 68.3 \\
\hline 2. & $\begin{array}{l}0.09\left(\mathrm{~m}, \mathrm{CH}_{2}\right) \\
1.23\left(\mathrm{t}, \mathrm{C}_{3}\right)\end{array}$ & $\begin{array}{l}2.12\left(\mathrm{~s}, \mathrm{CH}_{3}\right) ; \\
7.38(\mathrm{t}, \mathrm{H}-4) ; \\
7.79(\mathrm{br}, \mathrm{H}-5) ; \\
8.52(\mathrm{br}, \mathrm{H}-3) ; \\
9.38(\mathrm{br}, \mathrm{H}-6)\end{array}$ & $\begin{array}{l}10.4\left(\mathrm{CH}_{2}\right) \\
17.8\left(\mathrm{CH}_{3}\right)\end{array}$ & $\begin{array}{l}15.2\left(\mathrm{CH}_{3}\right) ; 120.1 \\
(\mathrm{C}-5) ; 122.5(\mathrm{C}-3) \\
134.9(\mathrm{C}-4) ; 147.8 \\
(\mathrm{C}-6) ; 148.5(\mathrm{C}-2) \\
149.5(\mathrm{C}=\mathrm{N})\end{array}$ & 66.6 \\
\hline 3. & $\begin{array}{l}0.44\left(\mathrm{br}, \mathrm{CH}_{2}\right) ; \\
1.72\left(\mathrm{br}, \mathrm{CH}_{3}\right)\end{array}$ & $\begin{array}{l}2.64\left(\mathrm{~s}, \mathrm{CH}_{3}\right) \\
7.51(\mathrm{~d}, \mathrm{H}-4) \\
7.73(\mathrm{~s}, \mathrm{H}-3) \\
7.82(\mathrm{~s}, \mathrm{H}-5)\end{array}$ & $\begin{array}{l}1.11\left(\mathrm{CH}_{2}\right) \\
18.2\left(\mathrm{CH}_{3}\right)\end{array}$ & $\begin{array}{l}12.1\left(\mathrm{CH}_{3}\right) ; 126.1 \\
(\mathrm{C}-4) ; 126.7(\mathrm{C}-3) \\
127.0(\mathrm{C}-5) ; 140.0 \\
(\mathrm{C}-2) ; 152.5(\mathrm{C}=\mathrm{N})\end{array}$ & 70.0 \\
\hline 4. & $\begin{array}{l}0.85\left(\mathrm{~m}, \mathrm{CH}_{2}\right) \\
1.33\left(\mathrm{t}, \mathrm{C}_{3}\right)\end{array}$ & $\begin{array}{l}2.18\left(\mathrm{~s}, \mathrm{CH}_{3}\right) ; \\
6.94(\mathrm{br}, \mathrm{H}-4) ; \\
7.29(\mathrm{~s}, \mathrm{H}-3) \\
7.38(\mathrm{~s}, \mathrm{H}-5)\end{array}$ & $\begin{array}{l}13.0\left(\mathrm{CH}_{2}\right) ; \\
18.2\left(\mathrm{CH}_{3}\right)\end{array}$ & $\begin{array}{l}14.0\left(\mathrm{CH}_{3}\right) ; 126.1 \\
(\mathrm{C}-4) ; 126.7(\mathrm{C}-3) ; \\
130.1(\mathrm{C}-5) ; 141.4 \\
(\mathrm{C}-2) ; 152.5(\mathrm{C}=\mathrm{N})\end{array}$ & 65.0 \\
\hline 5. & $\begin{array}{l}0.91\left(\mathrm{br}, \mathrm{CH}_{2}\right) \\
1.33\left(\mathrm{br}, \mathrm{CH}_{3}\right)\end{array}$ & $\begin{array}{l}2.18\left(\mathrm{~s}, \mathrm{CH}_{3}\right) ; \\
6.59(\mathrm{~d}, \mathrm{H}-4) ; \\
7.41(\mathrm{~s}, \mathrm{H}-3) ; \\
7.54(\mathrm{br}, \mathrm{H}-5)\end{array}$ & $\begin{array}{l}13.9\left(\mathrm{CH}_{2}\right) ; \\
17.6\left(\mathrm{CH}_{3}\right)\end{array}$ & $\begin{array}{l}11.1\left(\mathrm{CH}_{3}\right) ; 109.0 \\
(\mathrm{C}-4) ; 111.1(\mathrm{C}-3) \\
142.5(\mathrm{C}-5) ; 147.8 \\
(\mathrm{C}-2) ; 150.9(\mathrm{C}=\mathrm{N})\end{array}$ & 70.0 \\
\hline 6. & $\begin{array}{l}0.88\left(\mathrm{~m}, \mathrm{CH}_{2}\right) ; \\
1.26\left(\mathrm{br}, \underline{\mathrm{C}}_{3}\right)\end{array}$ & $\begin{array}{l}2.15\left(\mathrm{~s}, \mathrm{CH}_{3}\right) ; \\
6.27(\mathrm{br}, \mathrm{H}-4) ; \\
6.53(\mathrm{br}, \mathrm{H}-3) ; \\
7.29(\mathrm{br}, \mathrm{H}-5)\end{array}$ & $\begin{array}{l}11.5\left(\mathrm{CH}_{2}\right) ; \\
17.6\left(\mathrm{CH}_{3}\right)\end{array}$ & $\begin{array}{l}12.1\left(\mathrm{CH}_{3}\right) ; 108.3 \\
(\mathrm{C}-4) ; 111.1(\mathrm{C}-3) \\
142.6(\mathrm{C}-5) ; 148.8 \\
(\mathrm{C}-2) ; 151.7(\mathrm{C}=\mathrm{N})\end{array}$ & 63.7 \\
\hline 7. & $\begin{array}{l}0.09\left(\mathrm{~m}, \mathrm{CH}_{2}\right) \\
1.17\left(\mathrm{t}, \mathrm{CH}_{3}\right)\end{array}$ & $\begin{array}{l}7.29(\mathrm{~s}, \mathrm{CH}) ; 7.41 \\
(\mathrm{t}, \mathrm{H}-4) ; 7.60 \text { (d, H- } \\
3) ; 7.70(\mathrm{~d}, \mathrm{H}-5)\end{array}$ & $\begin{array}{l}0.92\left(\mathrm{CH}_{2}\right) \\
18.5\left(\mathrm{CH}_{3}\right)\end{array}$ & $\begin{array}{l}126.4(\mathrm{C}-4) ; 131.4 \\
(\mathrm{C}-3) ; 131.6(\mathrm{C}-5) \\
139.8(\mathrm{C}-2) ; 150.4 \\
(\mathrm{C}=\mathrm{N})\end{array}$ & 70.4 \\
\hline 8. & $\begin{array}{l}0.09\left(\mathrm{~m}, \mathrm{CH}_{2}\right) \\
1.26\left(\mathrm{br}, \underline{\mathrm{CH}}_{3}\right)\end{array}$ & $\begin{array}{l}7.35(\mathrm{~s}, \mathrm{CH}) ; 7.48 \\
(\mathrm{t}, \mathrm{H}-4) ; 7.54(\mathrm{~d}, \mathrm{H}- \\
3) ; 7.79(\mathrm{~d}, \mathrm{H}-5)\end{array}$ & $\begin{array}{l}0.89\left(\mathrm{CH}_{2}\right) \\
18.0\left(\mathrm{CH}_{3}\right)\end{array}$ & $\begin{array}{l}125.7(\mathrm{C}-4) ; 131.4 \\
(\mathrm{C}-3) ; 131.6(\mathrm{C}-5) \\
139.9(\mathrm{C}-2) ; 151.8 \\
(\mathrm{C}=\mathrm{N})\end{array}$ & 71.1 \\
\hline 9. & $\begin{array}{l}0.95\left(\mathrm{~m}, \mathrm{CH}_{2}\right) \\
1.20\left(\mathrm{br}, \mathrm{CH}_{3}\right)\end{array}$ & $\begin{array}{l}6.49(\mathrm{br}, \mathrm{H}-4) ; 7.29 \\
(\mathrm{~s}, \mathrm{CH}) ; 7.41(\mathrm{br}, \mathrm{H} \\
-3) ; 7.73(\mathrm{br}, \mathrm{H}-5)\end{array}$ & $\begin{array}{l}0.81\left(\mathrm{CH}_{2}\right) ; \\
18.3\left(\mathrm{CH}_{3}\right)\end{array}$ & $\begin{array}{l}111.2(\mathrm{C}-4) ; 112.4 \\
(\mathrm{C}-3) ; 144.2(\mathrm{C}-5) \\
146.9(\mathrm{C}-2) ; 152.0 \\
(\mathrm{C}=\mathrm{N})\end{array}$ & 67.9 \\
\hline 10. & $\begin{array}{l}0.12\left(\mathrm{~m}, \mathrm{CH}_{2}\right) \\
1.20\left(\mathrm{t}, \mathrm{CH}_{3}\right)\end{array}$ & $\begin{array}{l}6.40(\mathrm{br}, \mathrm{H}-4) ; 7.29 \\
(\mathrm{~s}, \mathrm{CH}) ; 7.51(\mathrm{br}, \mathrm{H} \\
-3) ; 7.76(\mathrm{br}, \mathrm{H}-5)\end{array}$ & $\begin{array}{l}0.81\left(\mathrm{CH}_{2}\right) ; \\
18.2\left(\mathrm{CH}_{3}\right)\end{array}$ & $\begin{array}{l}111.7(\mathrm{C}-4) ; 112.1 \\
(\mathrm{C}-3) ; 144.3(\mathrm{C}-5) \\
146.6(\mathrm{C}-2) ; 155.0 \\
(\mathrm{C}=\mathrm{N})\end{array}$ & 71.0 \\
\hline
\end{tabular}

*Complexes no. as in Table I 
This gets support from the significant upfield shifting of the positions of methyl and $\mathrm{CH}$ signals of the ligand moiety. However, no appreciable shift was observed in the position of ring protons of the functionalized oximes, ruling out the possibility of coordination through the hetero $(\mathrm{N}, \mathrm{O}$ or $\mathrm{S}$ ) atom. 'H NMR spectra of these derivatives exhibit characteristic peaks and peak multiplicities for $\mathrm{Et}-\mathrm{Al}$ and ligand protons $/ 6,29,30 /$. In the ${ }^{1} \mathrm{H}$ NMR spectra of the complexes, the $\mathrm{CH}_{2}$ and $\mathrm{CH}_{3}$ protons of $\mathrm{Et}-\mathrm{Al}$ group appear in the range 80.06$0.95 \mathrm{ppm}$ and $\delta 1.07-1.72 \mathrm{ppm}$, respectively. The methyl as well as $\mathrm{CH}$ proton resonance of ligands are shielded on complexation. Shielding effect is greater in 1:2 complexes as compared to 1:1 complexes. Other resonances are almost unaffected and appear at their expected positions (Table III).

\section{${ }^{13}$ C NMR Spectra}

The mode of bonding suggested above has also been confirmed by the ${ }^{13} \mathrm{C}$ NMR spectral data (Table III). In the ${ }^{13} \mathrm{C}$ NMR spectra of these complexes the $\mathrm{CH}_{2}$ and $\mathrm{CH}_{3}$ carbon signals of $\mathrm{EtAl}$ group appear in the ranges $\delta 0.81-13.9 \mathrm{ppm}$ and $\delta 17.6-18.5 \mathrm{ppm}$, respectively. The $\mathrm{C}=\mathrm{N}$ carbon resonance of ligands is shielded on complexation as compared to the free oximes, which suggests that there may be coordination of aluminium through nitrogen atom as evinced by IR spectra. The carbon signals due to heterocyclic rings of the functionalized oximes have been observed at their expected positions and do not show any appreciable shift. This further supports the view that heteroatom $(\mathrm{N}, \mathrm{O}$ or $\mathrm{S})$ does not take part in bonding.

\section{FAB Mass Spectra}

The FAB mass spectra of two representative compounds, $\mathrm{Et}_{2} \mathrm{Al}\left\{\mathrm{ON}=\mathrm{C}\left(\mathrm{CH}_{3}\right) \mathrm{C}_{4} \mathrm{H}_{3} \mathrm{~S}-2\right)$ and EtAl $\left\{\mathrm{ON}=\mathrm{C}\left(\mathrm{CH}_{3}\right) \mathrm{C}_{5} \mathrm{H}_{4} \mathrm{~N}-2\right\}_{2}$, indicate that the largest number of mass ion peaks are present in both the monomeric and dimeric form. However, the FAB mass spectra of the above compounds show fragmentation ion peaks with higher $\mathrm{m} / 4$ than the molecular ion, suggesting that the complexes are associated in the solid state.

The characteristic fragmentation ion peaks of the compounds $\mathrm{Et}_{2} \mathrm{Al}\left\{\mathrm{ON}=\mathrm{C}\left(\mathrm{CH}_{3}\right) \mathrm{C}_{4} \mathrm{H}_{3} \mathrm{~S}-2\right)$ and EtAI $\left\{\mathrm{ON}=\mathrm{C}\left(\mathrm{CH}_{3}\right) \mathrm{C}_{5} \mathrm{H}_{4} \mathrm{~N}-2\right\}_{2}$ are summarized in Schemes I and 2.

Scheme 1: FAB Mass spectral data for $\mathrm{Et}_{2} \mathrm{AI}\left\{\mathrm{ON}=\mathrm{C}\left(\mathrm{CH}_{3}\right) \mathrm{C}_{4} \mathrm{H}_{3} \mathrm{~S}-2\right)$

\begin{tabular}{|l|l|}
\hline $\mathrm{m} / \mathrm{e}$ & \\
\hline 488 & $\mathrm{Et}_{2} \mathrm{Al}\left\{\mathrm{ON}=\mathrm{C}\left(\mathrm{CH}_{3}\right) \mathrm{C}_{4} \mathrm{H}_{3} \mathrm{~S}-2\right\} \cdot\left(\mathrm{C}_{4} \mathrm{H}_{3} \mathrm{~S}-2\right)_{3} \cdot \mathrm{CH}_{2}$ \\
391 & $\mathrm{Et}_{2} \mathrm{Al}\left\{\mathrm{ON}=\mathrm{C}\left(\mathrm{CH}_{3}\right) \mathrm{C}_{4} \mathrm{H}_{3} \mathrm{~S}-2\right\} .\left(\mathrm{C}_{4} \mathrm{H}_{3} \mathrm{~S}-2\right)_{2}$ \\
308 & $\mathrm{Et}_{2} \mathrm{Al}\left\{\mathrm{ON}=\mathrm{C}\left(\mathrm{CH}_{3}\right) \mathrm{C}_{4} \mathrm{H}_{3} \mathrm{~S}-2\right\} . \mathrm{C}_{4} \mathrm{H}_{3} \mathrm{~S}-2$ \\
295 & $\mathrm{Et}_{2} \mathrm{Al}\left\{\mathrm{ON}=\mathrm{C}\left(\mathrm{CH}_{3}\right) \mathrm{C}_{4} \mathrm{H}_{3} \mathrm{~S}-2\right\} . \mathrm{C}_{3} \mathrm{H}_{2} \mathrm{~S}$ \\
232 & $\mathrm{Et}_{2} \mathrm{Al}\left\{\mathrm{ON}=\mathrm{C}\left(\mathrm{CH}_{3}\right) \mathrm{C}_{4} \mathrm{H}_{3} \mathrm{~S}-2\right\} . \mathrm{C}_{3}$ \\
220 & $\mathrm{Et}_{2} \mathrm{Al}\left\{\mathrm{ON}=\mathrm{C}\left(\mathrm{CH}_{3}\right) \mathrm{C}_{4} \mathrm{H}_{3} \mathrm{~S}-2\right\} \cdot \mathrm{C}_{2}$ \\
157 & $\mathrm{Et}_{2} \mathrm{Al}\left\{\mathrm{ON}=\mathrm{C}\left(\mathrm{CH}_{3}\right) \mathrm{CS}\right\}$ \\
142 & $\mathrm{CH}_{2} \mathrm{Al}\left\{\mathrm{ON}=\mathrm{C}\left(\mathrm{CH} \mathrm{CH}_{3}\right) \mathrm{CS}\right\}$ \\
124 & $\mathrm{CH}_{2} \mathrm{Al}\{\mathrm{N}=\mathrm{C}(\mathrm{CH}) \mathrm{CS}\}$ \\
\hline
\end{tabular}


Scheme 2: FAB Mass spectral data for $\operatorname{EtAl}\left\{\mathrm{ON}=\mathrm{C}\left(\mathrm{CH}_{3}\right) \mathrm{C}_{5} \mathrm{H}_{4} \mathrm{~N}-2\right\}_{2}$

\begin{tabular}{|l|l|}
\hline $\mathrm{m} / 4$ & \\
\hline 460 & EtAl $\left\{\mathrm{ON}=\mathrm{C}\left(\mathrm{CH}_{3}\right) \mathrm{C}_{5} \mathrm{H}_{4} \mathrm{~N}-2\right\}_{2} \cdot \mathrm{C}_{5} \mathrm{H}_{4} \mathrm{~N} \cdot \mathrm{C}_{3} \mathrm{H}_{6} \mathrm{~N}$ \\
443 & EtAl $\left\{\mathrm{ON}=\mathrm{C}\left(\mathrm{CH}_{3}\right) \mathrm{C}_{5} \mathrm{H}_{4} \mathrm{~N}-2\right\}_{2} \cdot \mathrm{C}_{5} \mathrm{H}_{4} \mathrm{~N} \cdot \mathrm{C}_{3} \mathrm{H}_{3}$ \\
391 & EtAl $\left\{\mathrm{ON}=\mathrm{C}\left(\mathrm{CH}_{3}\right) \mathrm{C}_{5} \mathrm{H}_{4} \mathrm{~N}-2\right\}_{2} \cdot \mathrm{C}_{5} \mathrm{H}_{5}$ \\
307 & EtAl $\left\{\mathrm{ON}=\mathrm{C}\left(\mathrm{CH}_{3}\right) \mathrm{C}_{3}\right\}_{2} \cdot \mathrm{C}_{5} \mathrm{H}_{5}$ \\
242 & EtAl $\left\{\mathrm{ON}=\mathrm{C}\left(\mathrm{CH}_{3}\right) \mathrm{C}_{3}\right\}_{2}$ \\
165 & $\mathrm{Al}\left\{\mathrm{ON}=\mathrm{C}\left(\mathrm{CH}_{3}\right) \mathrm{C}\right\}_{2}$ \\
153 & $\mathrm{Al}\left\{\mathrm{ON}=\mathrm{C}\left(\mathrm{CH}_{3}\right) \mathrm{C}\right\}\left\{\mathrm{ON}=\mathrm{C}\left(\mathrm{CH}_{3}\right)\right\}$ \\
136 & $\mathrm{Al}\left\{\mathrm{ON}=\mathrm{C}\left(\mathrm{CH}_{3}\right) \mathrm{C}\right\}\left\{\mathrm{N}=\mathrm{C}\left(\mathrm{CH}_{2}\right)\right\}$ \\
120 & $\left.\mathrm{Al}\left\{\mathrm{N}=\mathrm{C}\left(\mathrm{CH}_{3}\right) \mathrm{C}\right\} \mathrm{N}=\mathrm{C}\left(\mathrm{CH} \mathrm{H}_{2}\right)\right\}$ \\
107 & $\mathrm{Al}\left\{\mathrm{N}=\mathrm{C}\left(\mathrm{CH}_{3}\right) \mathrm{C}\right\}\{\mathrm{N}=\mathrm{CH}\}$ \\
\hline
\end{tabular}

\section{${ }^{27}$ Al NMR Spectra}

${ }^{27} \mathrm{Al}$ NMR spectra of these complexes exhibit a broad signal at 63.7-71.1 ppm, suggesting pentacoordination around aluminium(III) atom /8,31,32/.

\section{CONCLUSION}

Attempts to get good quality crystals of at least one representative compound for single crystal X-ray diffraction studies were not successful.

It is interesting to mention here that single crystal structure of an oximato complex, [(acac) $\left.{ }_{2} \mathrm{Zr}\left\{\mathrm{ON}=\mathrm{C}(\mathrm{Me}) \mathrm{C}_{5} \mathrm{H}_{4} \mathrm{~N}-2\right\}_{2}\right]$, indicates clearly that the oximato ligands bind $\mathrm{Zr}$ atom via $\mathrm{N}$ and $\mathrm{O}$ in a dihepto ( $\eta 2-\mathrm{N}, \mathrm{O})$ manner $/ 33 /$.

In view of the above studies as well as the observed dimeric nature of $1: 1$ and monomeric nature of 1:2 complexes, and the absence of the coordination through hetero $(\mathrm{N}, \mathrm{O}$ or $\mathrm{S})$ atom, the following plausible structures may be proposed tentatively for the types of derivatives ( $2 a$ and $2 b$ ).

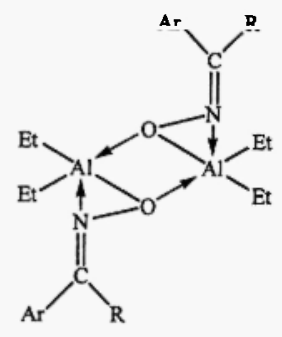

(2a)

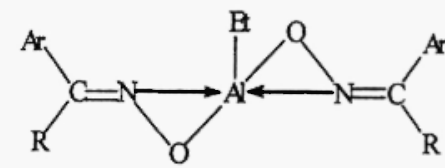

(2b)

Fig.: Proposed structures for $\mathrm{Et}_{2} \mathrm{Al}\{\mathrm{ON}=\mathrm{C}(\mathrm{R}) \mathrm{Ar}\}(2 \mathrm{a})$ and $\mathrm{EtAl}\{\mathrm{ON}=\mathrm{C}(\mathrm{R}) \mathrm{Ar}\}_{2}(2 \mathrm{~b})$

(where $\mathrm{R}=\mathrm{CH}_{3}$ or $\mathrm{H}$ and $\mathrm{Ar}=\mathrm{C}_{5} \mathrm{H}_{4} \mathrm{~N}-2, \mathrm{C}_{4} \mathrm{H}_{3} \mathrm{~S}-2, \mathrm{C}_{4} \mathrm{H}_{3} \mathrm{O}-2$ ) 


\section{ACKNOWLEDGEMENTS}

We are grateful to DST and UGC, New Delhi for financial support. One of the authors (A.K. Jain) is grateful to DST, New Delhi for the award of JRF.

\section{REFERENCES}

1. P. Sobota, S. Przybylak, J. Utko and L.B. Jerzykiewcz, Organometallics, 21, 3497 (2002).

2. J. Utko, S. Przybylak, L.B. Jerzykiewcz, K. Mierzwicki, Z. Lataijka and P. Sobota, Inorg. Chem., 42, 267 (2003).

3. M.D. Healy, J.W. Ziller and A.R. Barron, J. Am. Chem. Soc., 112, 2949 (1990).

4. C. Cui, H.W. Roesky, M. Noltemeyer, M.F. Lappert, H.-G. Schmidt and H. Hao, Organometallics, 18, 2256 (1999).

5. G.S. Hair, S.L. Battle, A. Decken, A.H. Cowley and R.A. Jones, Inorg. Chem., 39, 27 (2000).

6. W. Zheng, H. Hohmeister, N.C. Mosch-Zanetti, H.W. Roesky, M. Nolteemeyer and H.-G. Schmidt, Inorg. Chem., 40, 2363 (2001).

7. W. Uhl, J. Molter and B. Neumuller, Inorg. Chem., 40, 2011 (2001).

8. P.L. Gurian, L.K. Cheatham, J.W. Ziller and A.R. Barron, J. Chem. Soc. Dalton Trans., 1991, 1449.

9. J. Lewinski, J. Zachara, B. Mank and S. Pasynkiewcz, J. Organomet. Chem., 454, 5 (1993).

10. A.K. Jain, A. Gupta, R. Bohra and R.C. Mehrotra, Main Group Met. Chem., 26, 111 (2003).

11. A. Dhammani, R. Bohra and R.C. Mehrotra, Polyhedron, 15, 733 (1996).

12. N. Sharma, R.K. Sharma and R. Bohra, Main Group Met. Chem., 24, 781 (2001).

13. N. Sharma, R.K. Sharma, R. Bohra, J.E. Drake, M.B. Hursthouse and M.E. Light, J. Chem. Soc. Dalton Trans., 2002, 1631.

14. S. Nagar, R. Bohra and R.C. Mehrotra, Main Group Met. Chem., 25, 277 (2002).

15. N. Sharma, R.K. Sharma and R. Bohra, J. Chem. Research, (in press, 2003).

16. A.K. Jain, R. Bohra, R.C. Mehrotra, S. Nagar and S. Sharma, Heteroatom Chemistry, 14. 518 (2003).

17. N. Sharma, A.K. Jain, R.K. Sharma, R. Bohra, J.E. Drake, M.B. Hursthouse and M.E. Light, Polyhedron, 22, 2943 (2003).

18. (a) N.N. Gerasimchuk, E. Kuzmann, A. Buki, A. Vertes, L. Nagy and K. Burger, Inorg. Chim. Acta, 188(11), 45 (1991).

(b) K.V. Domasevich and N.N. Gerasimchuk, Russ. J. Inorg. Chem., 37(10), 3207 (1992).

(c) V.V. Monomareva, N.K. Dalley, X, Kou, N.N. Gerasimchuk and K.V. Domasevich, J. Chem. Soc. Dalton Trans., 1996, 2351.

19. (a) S.H. Davidson, Patent of USA \# 3780085 (1974).

(b) A. Hubele and M. Kuhne, Patent of USA \# 4063921 (1997).

20. (a) A.G. Ciba-Geigy, Patent of Austria \# 367268 (1982).

(b) A.G. Ciba-Geigy, Patent of Austria \# 127786 (1985). 
21. (a) H.A. Kirst, E.F. Szymanski, D.E. Doman, J.L. Occolowitz, N.D. Jones, M.O. Chaney, R.L. Hamill and M.M. Hoehn, J. Antibiot., 28(4), 286 (1975).

(b) H. Nakamura, Y. litaka, H. Sakakibara and H. Umezawa, J. Antibiot., 27(11), 894 (1974).

22. N. Auner and U. Klingebiel, Synthetic Methods of Organometallic and Inorganic Chemistry, 2, Georg Thieme Verlag Stuttgart, New York, 310 (1996).

23. (a) W. Huckel and M. Sachs, Ann. Chem., 498, 176 (1932).

(b) F. Nerdal and I. Huldschinsky, Chem. Ber., 86, 1005 (1953).

24. A.I. Vogel, Text Book of Quantitative Chemical Analysis, Longmans, London (Vth Edition) (1998).

25. E. Hecht, Z. Anorg. Allg. Chem., 626, 1642 (2000).

26. R. Bohra, A. Dhammani, R.K. Sharma and R.C. Mehrotra, Synth. React. Inorg. Met. Org. Chem., 36, $681(2001)$.

27. K. Serbest, I. Degirmencioglu, Ilinitials, pleasell Skarabocek and S. Guner, Trans. Met. Chem., 26, 232 (2001).

28. K.V. Domasevitch, N.N. Gerasimchuk and A. Mokhir, Inorg. Chem., 39, 1227 (2000).

29. J.E. Park, BH.-J. Bae, Y. Kim, J.T. Park and I.H. Such, Organometallics, 18, 1059 (1999).

30. S. Schulz and M. Nieger, Organometallics, 18, 315 (1999).

31. W. Ziemkowska, S. Pasynkiewicz and T. Skrok, Main Group Met. Chem., 21, 105 (1998).

32. R.M. Minyaev and A.G. Starikov, Russ. Chem. Bull., 48(7), 1225 (1999).

33. M. Pathak, R. Bohra, R.C. Mehrotra, I.-P. Lorenz and H. Piotrowski, Z. Anorg. Allg. Chem., 629, 2493 (2003). 
